\title{
Risk Factors for Mortality among Newborns with Neonatal Seizures
}

\author{
Osama Tanous ${ }^{1,2}$ Mohamad Watad ${ }^{1}$ Clari Felszer-Fisch ${ }^{3}$ Marina Peniakov ${ }^{3}$ Dan Miron ${ }^{1,2}$ \\ Raed Salim ${ }^{2,4}$
}

1 Pediatric Department A', Emek Medical Center, Afula, Israel

2 Rappaport Faculty of Medicine, Technion, Haifa, Israel

${ }^{3}$ Neonatal Intensive Care Unit, Emek Medical Center, Afula, Israel

${ }^{4}$ Department of Obstetrics and Gynecology, Emek Medical Center, Afula, Israel

Address for correspondence Raed Salim, MD, Department of Obstetrics and Gynecology, Emek Medical Center, Afula 18101, Israel (e-mail: salim_ra@clalit.org.il).

Neuropediatrics 2021;52:84-91.

\begin{abstract}
Keywords

- mortality

- prematurity

- neonatal seizures

- intracranial hemorrhage

Objective The aim of the study is to examine the incidence and risk factors for death among neonates who developed neonatal seizures (NS) in an ethnically distinctive community with high consanguinity rate in Israel.

Methods Retrospective study was conducted at a single institution on data between January 2001 and January 2016. All neonates diagnosed with NS developed up to age 28 days were included. Mortality was defined as death within the first year of life.

Results Of all 69,460 neonates born during the study period, 118 ( 1.7 per 1,000 live births) developed NS; 35 (29.7\%) died within the first year while 83 (70.3\%) survived. The leading causes of death were developmental brain malformation (31.4\%), genetic/ metabolic (20\%), hypoxic ischemic encephalopathy $(20 \%)$, intracranial hemorrhage $(11.4 \%)$ and infections (11.4\%). Any consanguinity between the parents was found in 18 and $14.6 \%$ among the survivors and deceased groups, respectively $(p=0.24)$. Developmental brain malformations that lead to death were present in 3.6 and $31.4 \%$ in the survivors and deceased groups, respectively $(p=0.001$; relative risk $8.70 ; 95 \%$ confidence interval 2.58-29.27). Stepwise backward logistic regression analysis revealed that developmental brain malformations $(p<0.0001)$, use of more than one antiepileptic medication $(p=0.006)$, and multiorgan failure $(p=0.004)$ were significant risk factors that predicted death.

Conclusion The results of the current study show that developmental brain malformations that cause NS were the leading risk factor for death.
\end{abstract}

\section{Introduction}

Seizures constitute the most frequent and distinctive manifestation of all neurological disturbances in the neonatal period and develop mainly in the first month of life. Neonatal seizures are considered a major risk for death, and survivors may develop neurological disability that can lead to adverse neurodevelopment later in life. ${ }^{1-4}$

received

July 16, 2019

accepted after revision

April 13, 2020

published online

October 22, 2020
Incidence of neonatal seizures ranges between two and five per 1,000 live births among term infants, while in preterm infants the incidence is tenfold higher. ${ }^{5-9}$ The large variation in the incidence of neonatal seizures reported can be explained by the different inclusion criteria used, ethnic diversity among the study population, the inconsistent methodologies used to define seizures, the lack of a clear description of clinical seizure presentations,

(c) 2020. Thieme. All rights reserved.

Georg Thieme Verlag KG,

Rüdigerstraße 14,

70469 Stuttgart, Germany
DOI https://doi.org/ 10.1055/s-0040-1712487. ISSN $0174-304 X$ 
particularly the subtle seizures, and differing referral patterns. $^{3,10}$

Mortality among neonates with seizures may reach $20 \%$ in the first year of life, particularly when accompanied with prematurity, low birthweight (LBW), low Apgar score, severe neonatal infection, severe hypoxic-ischemic encephalopathy (HIE), brain malformations, grade 3 or 4 intraventricular hemorrhage (IVH), and intracranial hemorrhage (ICH). ${ }^{11,12}$

It is essential to determine etiology to effectively treat the underlying cause and to offer a reasonable prognosis when counseling parents. ${ }^{3}$ Our institution is the most prominent health care providing institution in the northeast of Israel. The institution provides health care for two main ethnic communities, an Arab and Jewish population that vary considerably in certain parameters including consanguinity rate and maternal age. In view of this, we aimed in this study to examine incidence and risk factors for infant mortality related to neonatal seizures in these two distinctive culturally and ethnically diverse communities.

\section{Patients and Methods}

This is a retrospective cohort study conducted at Emek Medical Center, Afula, Israel, on data between January 2001 and January 2016. All neonates admitted with neonatal seizures to the neonatal intensive care unit (NICU), the nursery, the emergency unit, or pediatric wards during the study period were included.

Inclusion criteria included clinical seizures that developed within the first 28 days of life and documented by a physician; a pediatrician, a neonatologist or neurologist. In the NICU when a subclinical seizure was suspected electroencephalography (EEG) was performed for an average duration of 15 minutes. Newborns who succumbed to illness/death before investigations, had poor or no perinatal documentation; and neonates with jitteriness, tremor, and benign sleep myoclonus were excluded.

Data were obtained from the hospital discharge register with ICD-9 (International Classification of Disease, nineth revision) codes and crosschecked with the labor medical records. Evaluation included a thorough search for etiology, including detailed clinical history, maternal, obstetric, and perinatal parameters.

Data collected included maternal demographic, antenatal, intrapartum, and postpartum characteristics and outcomes. Neonatal variables collected included gender, ethnicity, year of birth, gestational age, and birthweight, age at the onset of seizure, type, duration, and frequency. A baseline detailed neurological examination was conducted by a neurologist at the onset of the seizure or during admission to the ICU and before discharge. The neurological exam was categorized to mild, moderate, severe in accordance to previous studies. ${ }^{13,14}$ Additionally, laboratory investigations that included blood glucose levels, sepsis screen, lumbar puncture results, serum electrolytes, EEG, neuroimaging results (ultrasonography, computerized tomography [CT], or magnetic resonance imaging [MRI]), viral serology screen, and screen for inborn errors of metabolism were extracted and documented.
Neonatal seizures were managed according to a standard protocol. When a genetic/metabolic disorder was suspected as in the case of seizures associated with poor feeding, prolonged lethargy, recurrent vomiting, family history of consanguinity, and/or neonatal seizure with early fetal and neonatal death, a primary metabolic work-up was undertaken that includes examining serum ammonia, serum lactate, arterial blood gas analysis, and urinary ketone. Further investigation was performed as needed. All children born at Emek Medical Center, like all children born in Israel underwent newborn screening according to the guidelines of the Ministry of Health. ${ }^{15}$ None of the diseases screened were present at our patients population. Etiology was grouped into eight categories as reported in the literature ${ }^{16,17}$ : HIE, ICH, infectious, vascular, genetic/metabolic (based on an accurate genetic diagnosis or a probable one due to different forms of dysmorphism, hypotonia, and visual or auditory disorders), developmental brain malformations (a wide range of cortical malformation including cortical dysplasia, hydrocephalus, not secondary to infection or hemorrhage, and more severe malformations like lissencephaly, holoprosencephaly, and varying degrees of hydranencephaly, based on ultrasound(US), CT, or MRI if conducted, not explained by other causes such as infections or vascular disorders), benign epilepsy syndromes, and transient metabolic disorders. Status epilepticus was defined as seizure for 30 minute or recurrent seizure that lasted more than 30 minutes without definite return to baseline neurological status between seizures. ${ }^{18}$ Multiorgan failure was defined as two or more organ dysfunction. An EEG was conducted in all children and interpreted by a pediatric neurologist. The EEG results were considered severely abnormal when there were significant asymmetries in the voltage or frequencies, low-voltage activity, or a permanent discontinuous activity; other abnormalities in the EEG were considered mild.

Neuroimaging studies' findings were considered severely abnormal when there was IVH grade 3 or 4 , intraparenchymal hemorrhage, periventricular hemorrhage, or brain malformations (- Supplementary Table 1, available online only). ${ }^{11,19}$

The primary outcome examined was death in the first year of life. Death was ascertained using the hospital, health care providers, and national mortality registry. For that reason the cohort study was divided to two groups, the survivors group and the deceased group, i.e., neonates who died within the first year.

Risk factors were categorized as those present before delivery (antenatal and intrapartum) and those related to neonatal outcome that were identified after delivery. The study protocol was approved by the local Institutional Review Board. For this type of study, signed consent was not required.

\section{Statistical Analysis}

Chi-square tests or Fisher's exact test where appropriate were performed to test differences in categorical variables between the two groups. Relative risk and 95\% confidence interval (CI) were computed. Student's $t$-test or Mann-Whitney test were performed to test differences in continuous data between the two groups and relative risk was estimated via logistic 
regression analysis. Backward stepwise regression using all significant variables was performed to determine multivariate risk factors for death.

\section{Results}

Of all 69,460 children born in our institution during the study period 118 (1.7 per 1,000 live births) were diagnosed with neonatal seizures. Of all these 118 neonates, 77 (65.3\%) were born at term and 41 (34.7\%) were preterm. The 77 term neonates with neonatal seizures comprised 1.2 per 1,000 term neonates ( 77 of 62,409 ) while the 41 preterm neonates comprised 5.8 per 1,000 live birth preterm neonates ( 41 of $7,051)$. Preterm neonates had 4.7 times the risk of experiencing neonatal seizures compared with term neonates (relative risk 4.7, 95\% CI 3.3-6.9, $p<0.0001$ ).

Any consanguinity between parents was found in 18 and $14.6 \%$ among the survivors and deceased groups, respectively $(p=0.24)$. Mean maternal age of all 118 neonates that composed the cohort study was 29.2 years and the mean gestational age at delivery was $35.9 \pm 5$ weeks. Mean birthweight was $2616 \pm 997 \mathrm{~g}, 38.5 \%$ were low birthweight $(<2,500 \mathrm{~g})$ and $17.4 \%$ were very low birthweight $(<1,500 \mathrm{~g})$. Additionally, 45 (38\%) neonates experienced a seizure in the first day of their life and 15 (12.7\%) experienced status epilepticus. The causes for neonatal seizures are presented in -Table 1.

\section{Risk Factors for Mortality}

Of all 118 newborns with neonatal seizures, 35 (29.7\%; 95\% CI: 22.2-38.4\%) died within the first year. The latter group comprised the "deaths" group while the other 83 neonates who survived for more than 1 year comprised the "survivors" group.
- Table 2 presents maternal demographic and obstetric, including antenatal, characteristics. No maternal demographic or obstetric characteristics were associated with neonatal death except for the presence of anomalies diagnosed on prenatal ultrasound $(p=0.001)$. Mode of delivery was equally divided between vaginal delivery and cesarean section (CS) with $81 \%$ of the CS of both groups being emergency section (-Table 2 ).

- Table 3 presents immediate neonatal outcomes. Neonates who were born small for gestational age were at higher risk for death within 1 year $(p=0.02)$. Additionally, Apgar scores of $<7$ at 1 minute $(p=0.04)$ and at 5 minutes $(p=0.006)$ were both associated with death within 1 year. Additionally, $60 \%$ of the neonates who died compared with $31 \%$ of those who survived required immediate resuscitation in the delivery ward $(p=0.004)$.

- Table 4 presents neonatal findings of the two groups after delivery. HIE was the leading cause for neonatal seizures overall, accounting for 24 (20\%) of all 118 neonates. When the relationship between the etiology of seizure and mortality was examined, the prevalence of developmental brain malformations was significantly higher in the death group (31.4\% compared with $3.6 \%, p=0.001)$. Benign epilepsy syndromes and unknown etiology were protective against death $(p<0.05)$. Furthermore, neonates who developed status epilepticus were at higher risk for death within 1 year $(p=0.001)$. Abnormal neurological exam at admission $(p=0.02)$, the use of more than one antiepileptic medication $(p=0.002)$, multiorgan failure $(p=0.001)$, and mechanical ventilation $(p=0.008)$ were also significantly more prevalent among the deceased neonates. Severely abnormal EEG was also significantly more common in the "death" group (16vs. 9.6\%, $p=0.001$; - Table 4).

Table 1 Etiology and mortality rate among newborns with neonatal seizures

\begin{tabular}{|l|l|l|l|l|}
\hline Seizure etiology & Deaths $(N=35)$ & Survivors $(N=83)$ & $p$-Value & Relative risk $(95 \%$ CI) \\
\hline Infectious $^{\text {a }}$ & $4(11.4)$ & $5(6.0)$ & 0.45 & $1.90(0.54-6.65)$ \\
\hline Hypoxic ischemic encephalopathy & $7(20.0)$ & $17(20.5)$ & 0.99 & $0.98(0.44-2.14)$ \\
\hline Intracranial hemorrhage & $4(11.4)$ & $16(19.3)$ & 0.42 & $0.46(0.16-1.30)$ \\
\hline Vascular & $1(2.9)$ & $3(4.6)$ & 0.99 & $0.79(0.09-7.34)$ \\
\hline Genetic/metabolic $^{\mathrm{b}}$ & $7(20.0)$ & $9(10.8)$ & 0.24 & $1.84(0.75-4.56)$ \\
\hline Developmental brain malformations $^{\mathrm{c}}$ & $11(31.4)$ & $3(3.6)$ & 0.001 & $8.70(2.58-29.27)$ \\
\hline Benign epilepsy syndrome $^{\mathrm{d}}$ & $0(0.0)$ & $10(12.0)$ & 0.03 & $0.11(0.01-1.77)$ \\
\hline Transient metabolic $^{\mathrm{e}}$ & $0(0.0)$ & $2(2.4)$ & 0.99 & $0.47(0.03-7.77)$ \\
\hline Unknown & $1(2.9)$ & $18(21.7)$ & 0.01 & $0.13(0.02-0.95)$ \\
\hline
\end{tabular}

Abbreviations: $\mathrm{Cl}$, confidence interval; GBS, group B Streptococcus.

${ }^{a}$ Two GBS meningitis, one pneumococcal meningitis, one Escherichia Coli meningitis, one Hemophilus Influenzae bacteremia, one GBS bacteremia, one Enterobacter and Candida albicans bacteremia, one Klebsiella pneumoniae bacteremia.

b One white matter abnormality presumed metabolic disorder, one VACTER (vertebral defects, anal atresia, cardiac defects, tracheo-esophageal fistula with esophageal atresia, and radial and renal dysplasia), one congenital lactic acidosis, two molybdenum cofactor deficiency, one trisomy 13 , one Coffin-Siris syndrome, one incontinentia pegmenti, one DI-GEORGE, the rest are different forms of dysmorphism, hypotonia, and visual or auditory disorders that lack a specific diagnosis.

'Two hydrocephalus, one hydranencephaly and polymicrogyria, one brain mass, one anencephaly and encephalocele, two holoprosencephaly, one severe brain dysplasia and agenesis of corpus callosum. One embryonal tumor in the posterior fossa originating from a pineal cyst. One choroid plexus cyst. One severe brain atrophy. One pachygyria and agenesis of corpus callosum. One anomaly in the vermis and cerebellum. One lissencephaly.

${ }^{\mathrm{d}}$ Six benign familial neonatal epilepsy, four benign nonfamilial neonatal epilepsy.

${ }^{\mathrm{e}}$ Two cases of hypoglycemia. 
Table 2 Maternal demographic and obstetric characteristics of the two groups of newborns who developed neonatal seizures

\begin{tabular}{|c|c|c|c|c|}
\hline Characteristic & Deaths $(N=35)$ & Survivors $(N=83)$ & $p$-Value & Relative risk $(95 \% \mathrm{Cl})$ \\
\hline \multicolumn{5}{|l|}{ Ethnicity } \\
\hline Arab & $22(62.9)$ & $50(60.2)$ & \multirow[t]{2}{*}{0.79} & $1.08(0.61-1.93)$ \\
\hline Jews & $13(37.1)$ & $33(39.8)$ & & 1.00 (reference) \\
\hline Maternal age, y & $29.6 \pm 5.6(29,25-33)$ & $29.0 \pm 6.3(28,24-33)$ & 0.67 & $1.02(0.95-1.09)$ \\
\hline Maternal age $>35$ y & $8(22.9)$ & $15(18.1)$ & 0.73 & $0.79(0.37-1.69)$ \\
\hline \multicolumn{5}{|l|}{ Conception } \\
\hline Spontaneous & $30(85.7)$ & $76(91.6)$ & \multirow[t]{4}{*}{0.19} & 1.00 (reference) \\
\hline Ovulation induction and & $0(0.0)$ & $1(1.2)$ & & $0.00(0.06-13.90)$ \\
\hline In vitro fertilization & $5(14.3)$ & $4(4.8)$ & & $1.96(0.89-3.22)$ \\
\hline Unknown & $0(0.0)$ & $2(2.4)$ & & 0.00 \\
\hline \multicolumn{5}{|l|}{ Consanguinity } \\
\hline None & $26(74.3)$ & $62(74.7)$ & \multirow[t]{4}{*}{0.24} & 1.00 (reference) \\
\hline First degree & $5(14.3)$ & $8(9.6)$ & & $1.30(0.57-2.42)$ \\
\hline Second degree & $0(0.0)$ & $7(8.4)$ & & $0.00(0.01-3.32)$ \\
\hline Unknown & $4(11.4)$ & $6(7.2)$ & & $1.35(0.54-2.57)$ \\
\hline Diabetes in pregnancy & $2(6.7)$ & $9(10.8)$ & 0.50 & $0.59(0.16-2.13)$ \\
\hline Placental abruption & $1(2.9)$ & $10(12.0)$ & 0.17 & $0.24(0.04-1.34)$ \\
\hline Pre-eclampsia & $1(2.9)$ & $2(2.4)$ & 0.99 & $1.19(0.11-12.66)$ \\
\hline Chorioamnionitis & $2(5.7)$ & $4(4.8)$ & 0.99 & $1.19(0.23-6.18)$ \\
\hline \multicolumn{5}{|l|}{ Prenatal ultrasound } \\
\hline Normal & $16(48.5)$ & $66(79.5)$ & 0.004 & 1.00 (reference) \\
\hline Brain anomaly & $8(24.2)$ & $8(9.6)$ & 0.02 & $2.56(1.33-4.95)$ \\
\hline Other anomalies & $9(27.3)$ & $9(10.8)$ & 0.01 & $2.56(1.35-4.55)$ \\
\hline Any anomaly & $17(51.5)$ & $17(20.5)$ & 0.001 & $2.56(1.47-4.46)$ \\
\hline Mean gestational age at delivery, (wk) & $35.0 \pm 5.5(37,31-39)$ & $36.3 \pm 4.7(39,35-39)$ & 0.20 & $0.95(0.88-1.03)$ \\
\hline \multicolumn{5}{|l|}{ Gestational age at delivery, (wk) } \\
\hline$<28$ wk & $6(17.1)$ & $7(8.4)$ & \multirow[t]{4}{*}{0.51} & $1.78(0.88-3.57)$ \\
\hline $28-36.6 w k$ & $5(14.3)$ & $10(12.0)$ & & $1.28(0.57-2.88)$ \\
\hline $34-36.6$ wk & $4(11.4)$ & $9(10.8)$ & & $1.18(0.48-2.91)$ \\
\hline$>37$ wk & $20(57.1)$ & $57(68.7)$ & & 1.00 (reference) \\
\hline Meconium amniotic fluid & $3(8.6)$ & $12(14.5)$ & 0.55 & $0.59(0.18-1.97)$ \\
\hline \multicolumn{5}{|l|}{ Mode of delivery } \\
\hline Vaginal & $13(37.1)$ & $46(55.4)$ & \multirow[t]{2}{*}{0.11} & 1.00 (reference) \\
\hline Cesarean & $22(62.9)$ & $37(44.6)$ & & $1.69(0.94-3.03)$ \\
\hline
\end{tabular}

Abbreviation: $\mathrm{Cl}$, confidence interval.

Note: Data are mean \pm standard deviation, (median, IQR), or $n(\%) ; p=0.02$, brain anomaly vs. normal; $p=0.01$, other anomaly vs. normal.

We performed a stepwise backward logistic regression analysis inserting into the model all significant variables that differed between the groups. The results revealed that developmental brain malformations as a cause of seizures $(p<0.0001)$, use of more than one antiepileptic medication $(p=0.006)$, and multiorgan failure $(p=0.004)$ were the only significant risk factors that predict neonatal death ( - Table 5).

Since consanguinity rate was expected to differ between the two ethnic communities that were included in the study, and consanguinity may affect outcome, we performed addi- tional analysis according to ethnicity. Of the 118 neonates 72 were Arabs (61\%) and 46 were Jews (39\%). The consanguinity rate among Arabs was significantly higher than among Jewish couples ( 26.4 vs. 2.2\%; $p=0.001$ ). Additionally, Arabic mothers were significantly younger than Jewish mothers (mean maternal age $27.7 \pm 5.3$ vs. $31.3 \pm 6.3 ; p=0.002$ ). After adjusting for factors that differ significantly between the groups, the results revealed that developmental brain malformations were significantly higher in Arab neonates compared with Jewish neonates (15.2 vs. $6.5 \%$; $p=0.03$ ). 
Table 3 Immediate neonatal outcomes of the two groups of newborn

\begin{tabular}{|c|c|c|c|c|}
\hline Characteristic & $\begin{array}{l}\text { Deceased neonates } \\
(N=35)\end{array}$ & $\begin{array}{l}\text { Survivors neonates } \\
(N=83)\end{array}$ & $p$-Value & $\begin{array}{l}\text { Relative risk } \\
(95 \% \mathrm{Cl})\end{array}$ \\
\hline \multicolumn{5}{|l|}{ Neonatal gender } \\
\hline Male & $23(65.7)$ & $49(59.0)$ & \multirow[t]{2}{*}{0.50} & $1.22(0.68-2.21)$ \\
\hline Female & $12(34.3)$ & $34(41.0)$ & & 1.00 (reference) \\
\hline Neonatal birthweight, $g^{a}$ & $\begin{array}{l}2,378 \pm 1,067 \\
(2,459 ; 1,510-2,856)\end{array}$ & $\begin{array}{l}2,733 \pm 947 \\
(3,060 ; 2,225-3,442)\end{array}$ & 0.06 & $0.95(0.91-0.99)$ \\
\hline Neonatal birthweight, $<2,500 \mathrm{~g}^{\text {a }}$ & $18 / 32(56.3)$ & $24 / 77(31.2)$ & 0.02 & $1.80(1.15-2.83)$ \\
\hline \multicolumn{5}{|l|}{ Neonatal birthweight, $\mathrm{g}^{\mathrm{a}}$} \\
\hline$<1,000$ & $6(17.1)$ & $7(8.4)$ & \multirow[t]{5}{*}{0.08} & $2.21(0.98-4.22)$ \\
\hline $1,000-1,500$ & $2(5.7)$ & $4(4.8)$ & & $1.60(0.47-5.42)$ \\
\hline $1,501-2,500$ & $10(28.6)$ & $13(15.7)$ & & $2.08(1.05-3.84)$ \\
\hline$>2,500$ & $14(40.0)$ & $53(63.9)$ & & 1.00 (reference) \\
\hline Missing & $3(8.6)$ & $6(7.2)$ & & $1.60(0.57-4.49)$ \\
\hline Apgar score at $1 \mathrm{~min}<7$ & $20(57.1)$ & 29 (34.9) & 0.04 & $1.32(1.02-1.72)$ \\
\hline Apgar score at $5 \mathrm{~min}<7$ & $16(45.7)$ & $16(19.3)$ & 0.006 & $1.56(1.08-2.24)$ \\
\hline Apgar score at $10 \mathrm{~min}<7$ & $5(14.3)$ & $8(9.6)$ & 0.52 & $1.48(0.52-4.22)$ \\
\hline Immediate resuscitation in the delivery ward & $21(60.0)$ & $26(31.1)$ & 0.004 & $1.45(1.10-1.92)$ \\
\hline Intubation in delivery ward & $21(60.0)$ & $34(41.0)$ & 0.06 & $1.26(0.98-4.84)$ \\
\hline
\end{tabular}

Abbreviations: $\mathrm{Cl}$, confidence interval; IQR, interquartile range.

Note: Data are mean \pm standard deviation, (median, IQR), or $n(\%)$.

${ }^{a}$ Three (8.6\%) and six (7.2\%) neonatal birthweights were missing from the deceased and survived groups, respectively.

\section{Discussion}

In this retrospective study we examined risk factors for infant mortality among neonates who developed seizures in ethnically diverse communities. The mortality rate found was almost 30\%. Preterm birth, LBW, Apgar score $<7$ after 1 and 5 minutes, need for immediate resuscitation in the delivery ward, mechanical ventilation, status epilepticus, and the need for more than one medication to control the seizures were all found to be associated with infant mortality. Additionally, abnormal neurological exam at admission to the NICU, severely abnormal EEG, and an abnormal brain imaging study were all found to be risk factors for mortality. After performing a stepwise backward logistic regression analysis, developmental brain malformations, use of more than one antiepileptic medication, and multiorgan failure were the only significant risk factors that predict neonatal death.

The developmental brain malformations found in our cohort, as shown in - Table 1, are considered major anatomical defects, and probably for that reason, led to the highest mortality rate (79\%, [11/14]) among all categories. The proportion of these significant malformations (11.8\%) was higher compared with the $4 \%$ found by Glass et al in their cohort. ${ }^{20}$ Though we have no precise explanation for this substantial difference, nevertheless, fewer terminations of pregnancy of prenatally diagnosed malformations or scarce prenatal care, or both, maybe the reason. The use of more than one antiepileptic medication and multiorgan failure represent a more severe clinical case and an end point toward death.
Overall, the incidence of neonatal seizures found was 1.7 per 1,000 live births, while preterm neonates were found to have almost five times the risk of experiencing seizures. Similar to previous reports, HIE followed by ICH was the most common reason for neonatal seizures. ${ }^{12,20,21}$

Although the overall incidence of neonatal seizures was similar to a rate of two to five per 1,000 live births reported in the literature, ${ }^{5-9}$ the $30 \%$ mortality rate found in the current study is almost double the rate compared with that reported by others. ${ }^{20,22-24}$ This may be explained by the characteristics of the study population and the high rate of consanguinity found in the current study compared with other reports. Consanguinity may lead to increased incidence of genetic/metabolic or developmental brain malformations, both of which were found to be associated with $51.4 \%$ of all causes of neonatal seizures among the deceased neonates.

For example, in the prospective study by Glass et al, ${ }^{20}$ brain malformations accounted for only $4 \%$ of the seizures and genetic/metabolic disorders for $3 \%$, in our study it accounted for 11.8 and $13.5 \%$, respectively. Comparable results were found in a smaller cohort with high consanguinity rate. ${ }^{25}$

The limitations of this study are those inherent in the use of retrospective databases. For that reason we were unable to collect thorough documentation regarding all variables that may affect outcome such as maternal education, socioeconomic status, the length, type, and number of seizures despite the use of multiple sources for identifying precise maternal and neonatal parameters and by manual inspection of each medical file. Additionally, the sample size in terms of certain 
Table 4 Neonatal findings of the two groups of newborns who developed neonatal seizures

\begin{tabular}{|c|c|c|c|c|}
\hline Characteristic & $\begin{array}{l}\text { Deceased neonates } \\
(N=35)\end{array}$ & $\begin{array}{l}\text { Survivors neonates } \\
(N=83)\end{array}$ & $p$-Value & $\begin{array}{l}\text { Relative risk } \\
(95 \% \mathrm{Cl})\end{array}$ \\
\hline \multicolumn{5}{|l|}{ Seizure onset } \\
\hline$\leq 1 \mathrm{~d}$ & $16(45.7)$ & $29(31.2)$ & 0.15 & 1.00 (reference) \\
\hline$>1 d$ & $19(54.3)$ & $64(68.8)$ & & $0.64(0.37-1.12)$ \\
\hline Status epilepticus & $11(31.4)$ & $4(4.8)$ & 0.001 & $6.52(2.23-19.09)$ \\
\hline \multicolumn{5}{|l|}{ Neurological exam ${ }^{a}$} \\
\hline Normal & $7(20.0)$ & $40(48.2)$ & \multirow[t]{4}{*}{0.02} & 1.00 (reference) \\
\hline Mild & $13(37.1)$ & $30(36.1)$ & & $2.03(0.89-4.61)$ \\
\hline Severe & $10(28.6)$ & $11(13.2)$ & & $3.20(1.41-7.24)$ \\
\hline Missing & $5(14.3)$ & $2(2.4)$ & & $4.80(2.09-10.98)$ \\
\hline \multicolumn{5}{|l|}{ Number of antiepileptic drugs } \\
\hline 0 & $0(0.0)$ & $6(7.2)$ & \multirow[t]{3}{*}{0.002} & $0.00(0.02-4.83)$ \\
\hline 1 & $23(65.7)$ & $70(90.9)$ & & 1.00 (reference) \\
\hline$>1$ & $12(34.3)$ & $7(9.1)$ & & $2.55(1.56-4.18)$ \\
\hline Mechanical ventilation & $27(77.1)$ & $42(50.6)$ & 0.008 & $1.52(1.15-2.01)$ \\
\hline Respiratory distress syndrome & $11(31.4)$ & $20(24.1)$ & 0.41 & $1.30(0.70-2.43)$ \\
\hline Multiorgan failure & $15(44.1)$ & $10(12.0)$ & 0.001 & $3.56(1.77-7.13)$ \\
\hline Mean length of admission & $25.9 \pm 22.5(23.0 ; 8.5-36.0)$ & $28.0 \pm 32.0(15.5 ; 9.0-31.3)$ & 0.60 & $1.00(0.98-1.01)$ \\
\hline Hospitalization $>7 \mathrm{~d}^{\mathrm{b}}$ & $26 / 33(78.8)$ & $69 / 80(86.3)$ & 0.32 & $0.91(0.75-1.11)$ \\
\hline \multicolumn{5}{|l|}{ Brain radiological exam ${ }^{c}$} \\
\hline Normal & $11(31.4)$ & $53(63.8)$ & \multirow[t]{3}{*}{0.003} & 1.00 (reference) \\
\hline Mild & $5(14.3)$ & $10(12.0)$ & & $1.94(0.79-4.75)$ \\
\hline Severe & $19(54.3)$ & $20(24.1)$ & & $2.83(1.51-5.31)$ \\
\hline \multicolumn{5}{|l|}{$\mathrm{EEG}^{\mathrm{d}}$} \\
\hline Normal & $20(57.1)$ & $23(27.7)$ & \multirow[t]{3}{*}{0.001} & 1.00 (reference) \\
\hline Mild & $9(25.7)$ & $52(62.6)$ & & $0.32(0.16-0.63)$ \\
\hline Severe & $6(17.1)$ & $8(9.6)$ & & $0.92(0.46-1.83)$ \\
\hline
\end{tabular}

Abbreviations: Cl, confidence interval; CT, computed tomography; EEG, electroencephalography; IQR, interquartile range; IVH, intraventricular hemorrhage; MRI, magnetic resonance imaging; US, ultrasound.

Note: Data are mean \pm standard deviation, (median, IQR), or $n(\%)$.

${ }^{a}$ The first neurological examination, evaluated according to gestational age, was classified into three categories; (1) Normal or mildly abnormal; (2) moderately abnormal, such as hypotonia/hypertonia, decreased muscle active movements, lethargy; (3) severely abnormal, such as flaccid, inactive, and coma.

${ }^{\mathrm{b}}$ Data regarding three and two cases in the survivors and deceased groups, respectively, were missing.

'Imaging studies US/CT/MRI findings were considered severely abnormal when there was IVH of degree three or four, intraparenchymal hemorrhage, periventricular hemorrhage, or brain malformations. Other abnormal radiological findings were considered mild.

${ }^{d}$ EEG was considered severely abnormal when there were significant asymmetries in the voltage or frequencies, low voltage activity, or a permanent discontinuous activity; other abnormalities in the EEG were considered mild.

Table 5 Significant risk factors for mortality following stepwise backward logistic regression

\begin{tabular}{|l|l|l|}
\hline Characteristic & $p$-Value & Relative risk (95\% CI) \\
\hline $\begin{array}{l}\text { Developmental brain } \\
\text { malformations }\end{array}$ & $<0.0001$ & $28.74(5.29-155.79)$ \\
\hline $\begin{array}{l}\text { Use of }>1 \text { antiepileptic } \\
\text { drug }\end{array}$ & 0.006 & $7.00(1.73-28.39)$ \\
\hline Multiorgan failure & 0.004 & $6.52(1.84-23.10)$ \\
\hline
\end{tabular}

Abbreviation: $\mathrm{Cl}$, confidence interval. outcomes examined may be too small to make definitive conclusions. This problem is common in retrospective studies generally and in studies of rare maloccurrences specifically. Finally, the fact that this study was performed at a single center with distinctive ethnically diverse communities might limit its generalizability. Nevertheless, a distinct advantage related to a single center study is the use of similar protocols for diagnosis and managing these neonates. Another limitation of the study is the use of clinical rather than electrographic criteria for the diagnosis of seizures. Literature indicates that only $50 \%$ of the clinical events are correctly classified as neonatal seizures, 
with poor interobserver agreement, ${ }^{26}$ and the challenge of identifying seizures in the neonatal period due to the presence of subclinical seizures identified only using EEG. ${ }^{4,27,28}$ Again this limitation is inherent to the retrospective nature of the study and the reliance on common practice. Nevertheless, the incidence of neonatal seizures found was similar to most published data.

Despite the improvement in perinatal care the mortality rate in newborns with seizures remains relatively high. Since different populations differ vastly in parameters such as ethnic background and rate of consanguinity, the degree of prenatal work-up and neonatal care may differ accordingly. It is therefore of utmost importance for neonatologists and neurologists to be familiar with precise risk factors for mortality when counseling parents. Additionally, recognizing risk factors for mortality may assist in early therapeutic and rehabilitative intervention. It still needs to be examined whether recognizing risk factors for mortality and early intervention can reduce the incidence of mortality.

\section{What's Known on This Subject}

Neonatal seizures may lead to infant mortality and morbidity. Etiology of seizures differs within different populations.

\section{What This Study Adds}

Among distinct ethnic communities the mortality rate in cases of neonatal seizures is almost double. This is probably related to distinguished risk factors including high consanguinity rate that may lead to precise risk factors, especially developmental brain malformations.

Financial Disclosure

All authors have indicated they have no financial relationships relevant to this article to disclose.

\section{Table of Contents Summary}

Characteristics of the community examined may determine the major risk factors for neonatal seizures and as a consequence negatively affect the neonatal mortality rate.

\section{Contributors' Statement Page}

O.T. and R.S. conceptualized and designed the study, drafted the initial manuscript, and reviewed and revised the manuscript. M.W. designed the data collection instruments, collected data, performed the initial analyses, and reviewed and revised the manuscript. C.F.-F., M.P., and D. M. conceptualized and designed the study, coordinated and supervised data collection, and critically reviewed the manuscript for important intellectual content. All authors approved the final manuscript as submitted and agree to be accountable for all aspects of the work.

\section{Conflict of Interest}

All authors have indicated they have no potential conflicts of interest to disclose.

\section{References}

1 Levene M. The clinical conundrum of neonatal seizures. Arch Dis Child Fetal Neonatal Ed 2002;86(02):F75-F77

2 Bassan H, Bental Y, Shany E, et al. Neonatal seizures: dilemmas in workup and management. Pediatr Neurol 2008;38(06):415-421

3 Vasudevan C, Levene M. Epidemiology and aetiology of neonatal seizures. Semin Fetal Neonatal Med 2013;18(04):185-191

4 Sands TT, McDonough TL. Recent advances in neonatal seizures. Curr Neurol Neurosci Rep 2016;16(10):92

5 Lanska MJ, Lanska DJ, Baumann RJ, Kryscio RJ. A population-based study of neonatal seizures in Fayette County, Kentucky. Neurology 1995;45(04):742

6 Lanska MJ, Lanska DJ. Neonatal seizures in the United States: results of the National Hospital Discharge Survey, 1980-1991. Neuroepidemiology 1996;15(03):117-125

7 Saliba RM, Annegers JF, Waller DK, Tyson JE, Mizrahi EM. Incidence of neonatal seizures in Harris County, Texas, 1992-1994. Am J Epidemiol 1999;150(07):763-769

8 Ronen GM, Penney S, Andrews W. The epidemiology of clinical neonatal seizures in Newfoundland: a population-based study. J Pediatr 1999;134(01):71-75

9 Glass HC, Pham TN, Danielsen B, Towner D, Glidden D, Wu YW. Antenatal and intrapartum risk factors for seizures in term newborns: a population-based study, California 1998-2002. J Pediatr 2009;154(01):24-28.e1

10 Murray DM, Boylan GB, Ali I, Ryan CA, Murphy BP, Connolly S. Defining the gap between electrographic seizure burden, clinical expression and staff recognition of neonatal seizures. Arch Dis Child Fetal Neonatal Ed 2008;93(03):F187-F191

11 Anand V, Nair PMC. Neonatal seizures: predictors of adverse outcome. J Pediatr Neurosci 2014;9(02):97-99

12 Heljic S, Uzicanin S, Catibusic F, Zubcevic S. Predictors of mortality in neonates with seizures; A prospective cohort study. Med Arh 2016;70(03):182-185

13 Legido A, Clancy RR, Berman PH, Legido A, Clancy RR, Berman PH. Neurologic outcome after electroencephalographically proven neonatal seizures. Pediatrics 1991;88(03):583-596

14 Pisani F, Facini C, Pelosi A, Mazzotta S, Spagnoli C, Pavlidis E. Neonatal seizures in preterm newborns: a predictive model for outcome. Eur J Paediatr Neurol 2016;20(02):243-251

15 Israeli Ministry of Health. Neonatal Screening. 2015. Available at: https://www.health.gov.il/English/Topics/Genetics/InfantScreening/Pages/default.aspx. Accessed May 14, 2020

16 Kang SK, Kadam SD. Neonatal seizures: impact on neurodevelopmental outcomes. Front Pediatr 2015;3:101

17 Olson DonaldM. Neonatal seizures. Neoreviews 2012;13(04): e213-e223

18 Pavlidis E, Spagnoli C, Pelosi A, Mazzotta S, Pisani F. Neonatal status epilepticus: differences between preterm and term newborns. Eur J Paediatr Neurol 2015;19(03):314-319

19 Pisani F, Sisti L, Seri S. A scoring system for early prognostic assessment after neonatal seizures. Pediatrics 2009;124(04): e580-e587

20 Glass HC, Shellhaas RA, Wusthoff CJ, et al; Neonatal Seizure Registry Study Group. Contemporary profile of seizures in neonates: a prospective cohort study. J Pediatr 2016;174:98-103.e1

21 Sheth RD, Hobbs GR, Mullett M. Neonatal seizures: incidence, onset, and etiology by gestational age. J Perinatol 1999;19(01): 40-43

22 Aziz A, Gattoo I, Aziz M, Rasool G. Clinical and etiological profile of neonatal seizures: a tertiary care hospital based study. Int J Res Med Sci 2015;3(09):2198-2203

23 Sabzehei MK, Basiri B, Bazmamoun H. The etiology, clinical type, and short outcome of seizures in newborns hospitalized in Besat Hospital/Hamadan/Iran. Iran J Child Neurol 2014;8(02):24-28

24 Ching Hu S, Hung KL, Chen HJ. Neonatal seizures: incidence, etiologies, clinical features and EEG findings in the neonatal intensive care unit. Epilepsy J 2017;3:3-5 
25 Badran EF, Masri AT, Hamamy H, Al-Qudah AA. Etiological and clinical profile of neonatal seizures in a highly consanguineous population. J Pediatr Neurol 2007;5(04):305-309

26 Malone A, Ryan CA, Fitzgerald A, Burgoyne L, Connolly S, Boylan GB. Interobserver agreement in neonatal seizure identification. Epilepsia 2009;50(09):2097-2101
27 McBride MC, Laroia N, Guillet R. Electrographic seizures in neonates correlate with poor neurodevelopmental outcome. Neurology 2000;55(04):506-513

28 Cross JH. Differential diagnosis of epileptic seizures in infancy including the neonatal period. Semin Fetal Neonatal Med 2013;18 (04):192-195 